\title{
Immunity to Haemophilus influenzae B and Pneumococcal vaccination among adult women with Turner Syndrome
}

\author{
Jakub Bukowczan ${ }^{1}$, Aaron Liew ${ }^{2-4}$, Graham Roberts ${ }^{5}$, Gavin Spickett ${ }^{1}$, Richard Quinton ${ }^{1,2}$. \\ Newcastle upon Tyne Hospitals NHS Foundation Trust ${ }^{1}$, Newcastle University Institute of Genetic Medicine ${ }^{2}$, Newcastle upon Tyne, United Kingdom, \\ National University of Ireland Galway (NUIG) ${ }^{3}$, Portiuncula University Hospital and Galway University Hospital, \\ Saolta University Health Care Group, Galway4, University College Cork, Ireland \& Swansea University, United Kingdom.
}

\section{Introduction}

- Turner Syndrome (TS) is associated with defective immunity, due to hemizygosity for important $X$ chromosomal loci, with greater risk of a variety of autoimmune diseases, but likely impaired recognition of real infectious threats.

- TS is associated with a higher overall morbidity and mortality than the general population, with respiratory and infectious diseases as two of the major causes.

- Haemophilus influenza type B (HiB) and pneumococcal $(P C)$ vaccination can potentially reduce morbidity and mortality, by preventing two specific respiratory infections.

- All patients in the Newcastle Adult Turner Syndrome Clinic who lack immunity to either HiB or PC at baseline receive vaccination in our TS clinic.

- However, the response rate following vaccination has not hitherto been examined.

\section{Methods}

- We prospectively examined the response rate to $\mathrm{HiB}$ and PC vaccination among a cohort of 100 consecutive adult women with TS.

- Patients with titres below these lower limits were considered to have inadequate immunity and vaccination was administered in primary care (Pneumovax or Menitorix).

- In our laboratory, the antibody titres at the protective range were $1-20$ and $20-200 \mathrm{mg} / \mathrm{L}$, for $\mathrm{HiB}$ and $\mathrm{PC}$ antigens, respectively.

\section{Results}

- A total of 96 eligible TS patients aged $\geq 18$ years were reported. The median age and BMI were $31.5(24.8-45.0)$ years and $26.1(23.2-30.7)$ $\mathrm{kg} / \mathrm{m}^{2}$, respectively.

- At baseline, $54.2 \%$ (52/96) and $18.8 \%$ (18/96) of patients had inadequate antibody response to HiB and PC vaccines, respectively (Figures $1 \mathrm{a} \& 2 \mathrm{a}$ ).

- Intriguingly, $27.5 \%(14 / 51)$ patients in the former and $38.9 \%(7 / 18)$ in the latter, also had a low lgM level $(<0.71)$ (Figures $1 \mathrm{~b} \& 2 \mathrm{~b})$.

- Furthermore, $7.8 \%(4 / 51)$ and $16.7 \%(3 / 18)$ had a low IgG level $(<5.8)$, respectively (Figures $1 \mathrm{c}$ \& 2c).

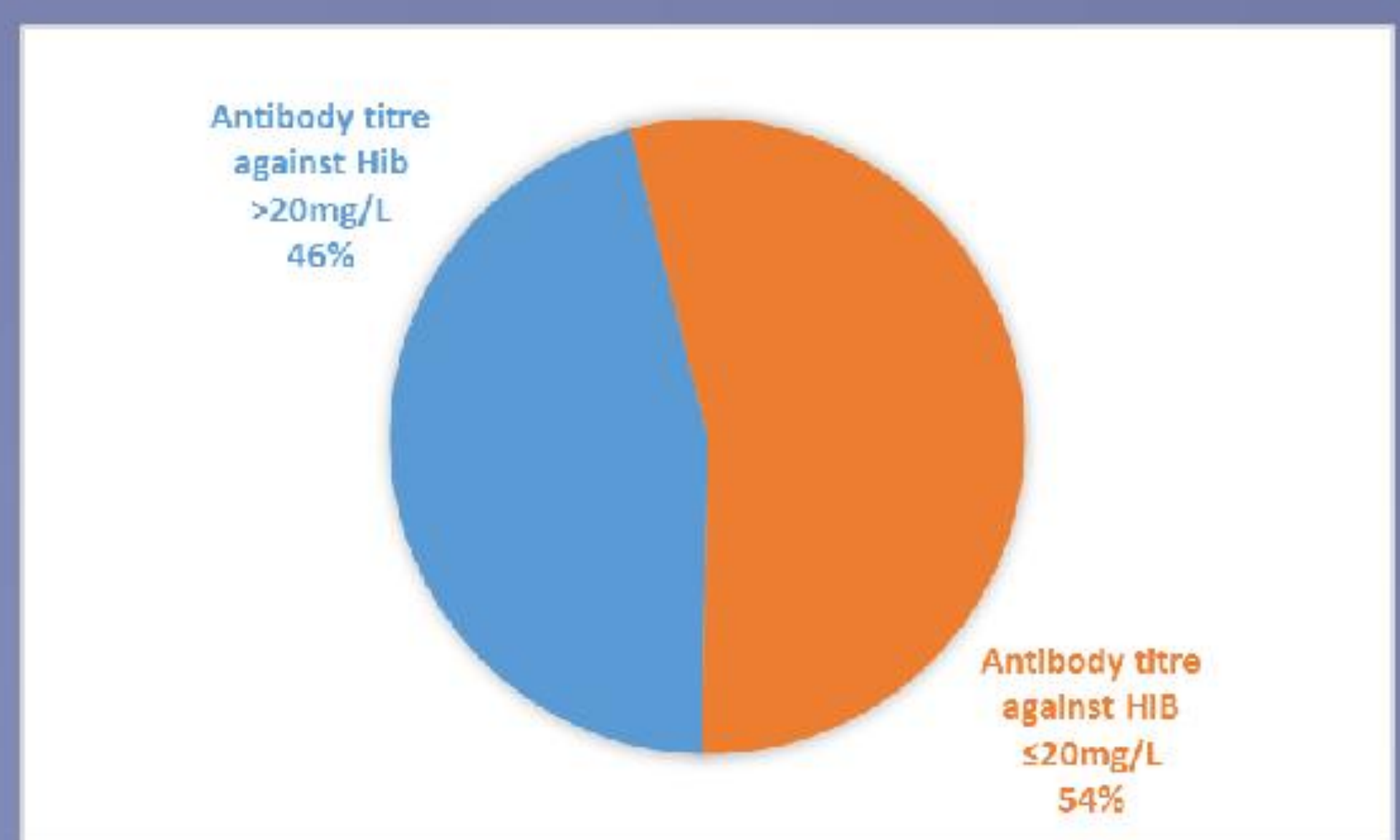

Figure 1a: HiB vaccine response.

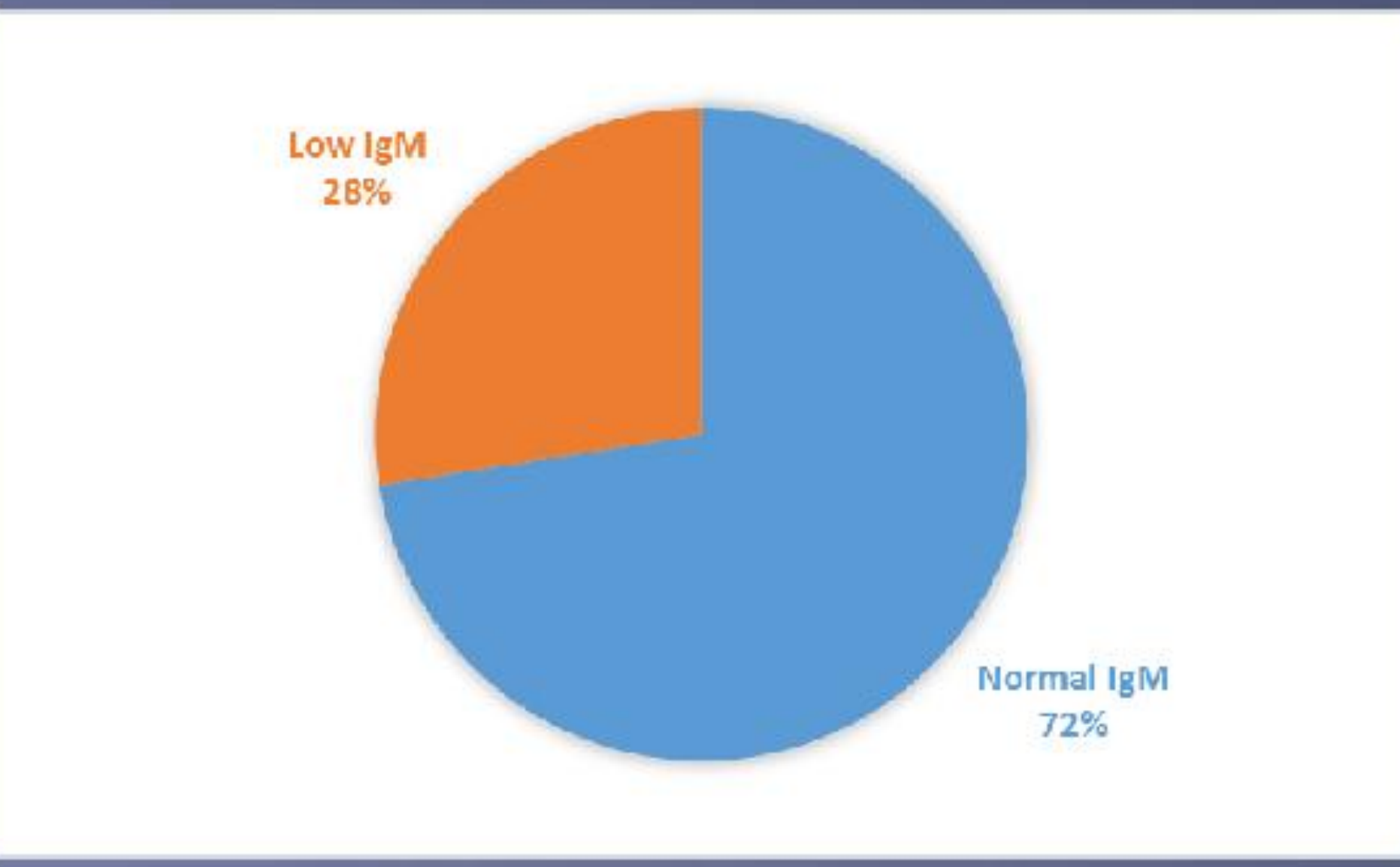

Figure 1b: Percentage of low lgG.

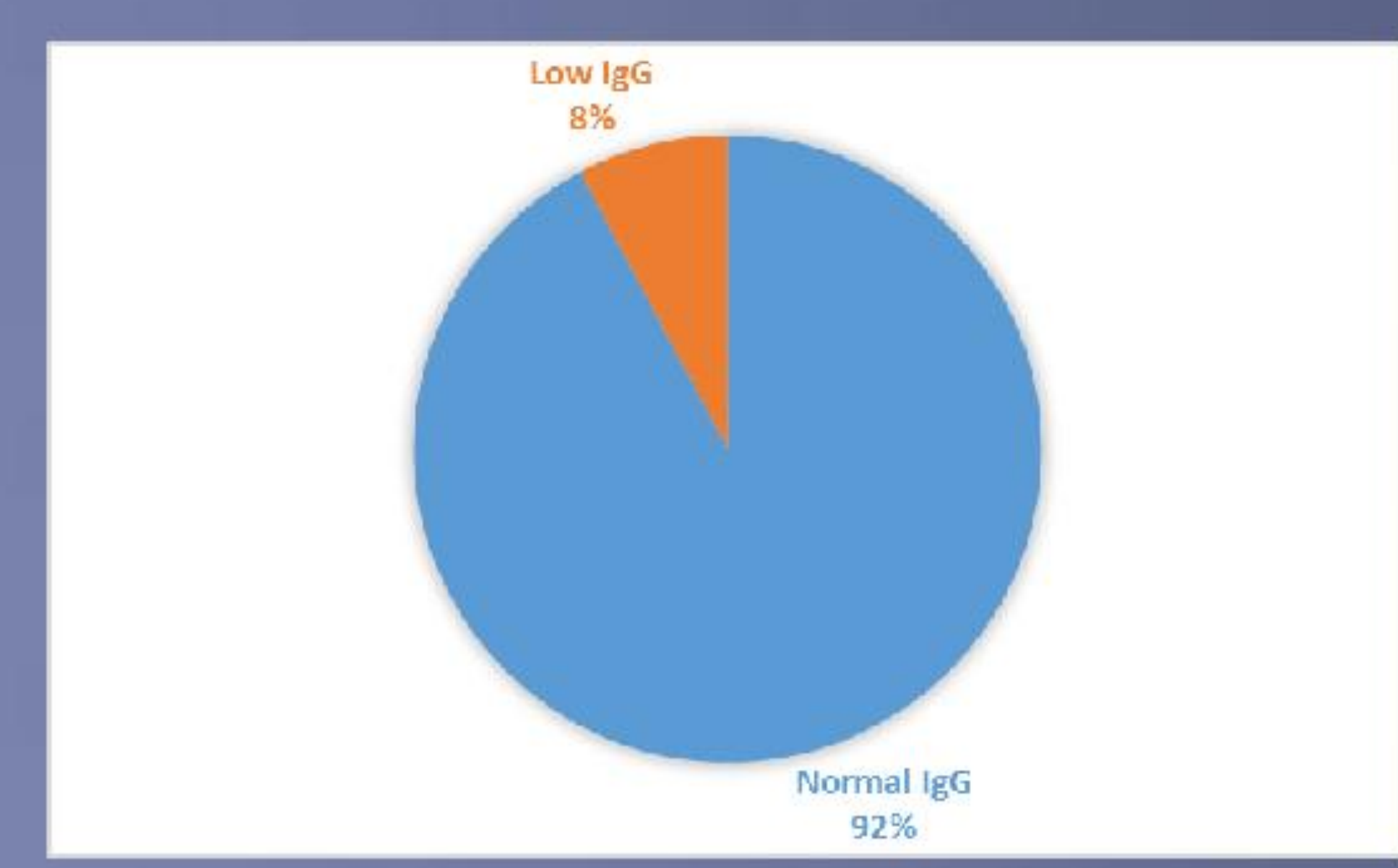

Figure 1c: Percentage of low lgM.

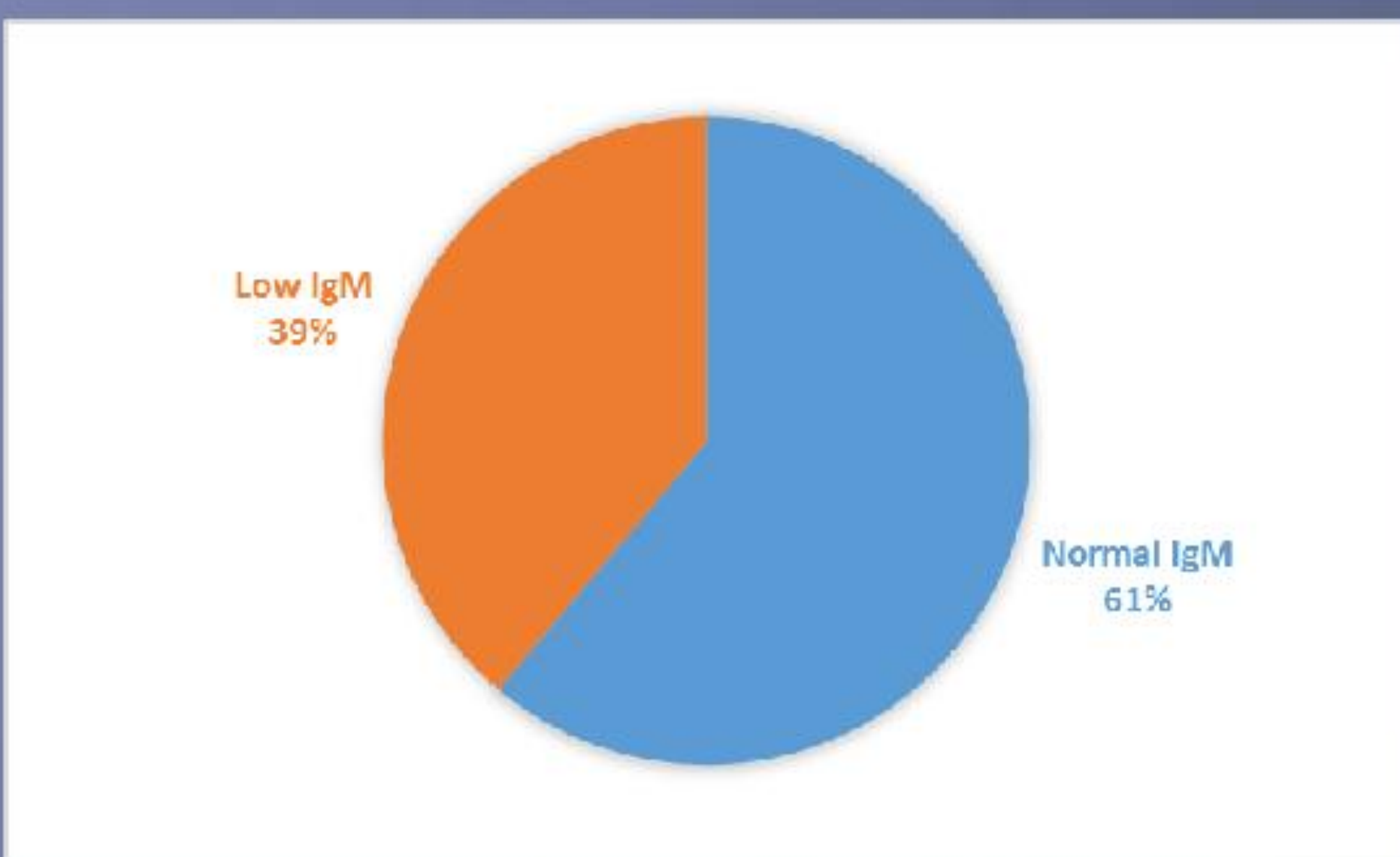

Figure 2b: Percentage of low lgG.

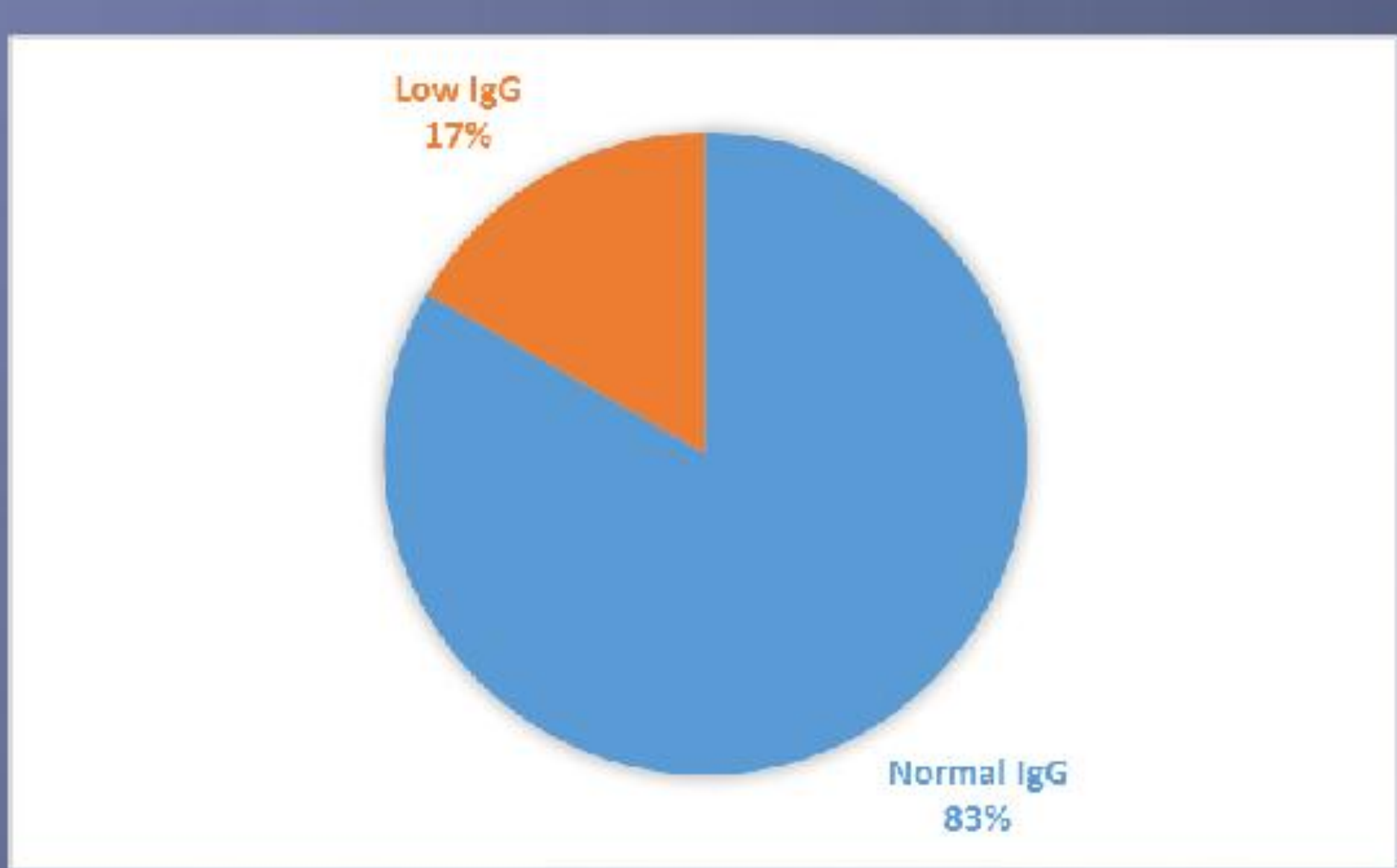

Figure 2c: Percentage of low IgM.

\section{Conclusion}

- Although it was not possible to identify a suitable control group, the prevalence of protective antibodies to HiB and PC among adult women with TS were markedly low in this cohort, hence, underpinning the importance of screening and vaccination to achieve protective titres, thereby potentially reducing related morbidity and mortality.

\section{Acknowledgement}

- This research is supported by the Exchange in Endocrinology Expertise (3E) Fellowship Grant awarded by the Section/Board of Endocrinology of the UEMS. 